\title{
Improving Awareness of Automated Actions using an Interactive Event Timeline
}

\author{
Y.-L. Betty Chang \\ ${ }^{1}$ Systems Design Engineering, \\ ${ }^{2}$ Games Institute \\ University of Waterloo \\ Waterloo, ON, Canada \\ betty.chang@uwaterloo.ca \\ Mylène Mengual \\ ${ }^{1}$ Systems Design Engineering, \\ ${ }^{2}$ Games Institute \\ University of Waterloo \\ Waterloo, ON, Canada \\ mylene.mengual@telecom- \\ bretagne.eu \\ Brian Parfett \\ ${ }^{1}$ Systems Design Engineering, \\ ${ }^{2}$ Games Institute \\ University of Waterloo \\ Waterloo, ON, Canada \\ btparfet@uwaterloo.ca \\ T.C. Nicholas Graham \\ chool of Computing \\ Kingston, ON, Canada \\ graham@cs.queensu.ca \\ Mark Hancock \\ Department of Management \\ Stacey D. Scott \\ ${ }^{1}$ Systems Design Engineering, \\ Games Institute \\ Waterloo, ON, Canada \\ stacey.scott@uwaterloo.ca \\ Queen's University
}

Permission to make digital or hard copies of part or all of this work for personal or classroom use is granted without fee provided that copies are not made or distributed for profit or commercial advantage and that copies bear this notice and the full citation on the first page. Copyrights for third-party components of this work must be honored. For all other uses, contact the Owner/Author.

Copyright is held by the owner/author(s).

ITS '13, Oct 06-09 2013, St Andrews, United Kingdom

ACM 978-1-4503-2271-3/13/10.

http://dx.doi.org/10.1145/2512349.2512398

\begin{abstract}
Digital tabletops provide an opportunity for automating complex tasks in collaborative domains involving planning and decision-making, such as strategic simulation in command and control. However, when automation leads to modification of the system's state, users may fail to understand how or why the state has changed, resulting in lower situation awareness and incorrect or suboptimal decisions. We present the design of an interactive event timeline that aims to improve situation awareness in tabletop systems that use automation. Our timeline enables exploration and analysis of automated system actions in a collaborative environment. We discuss two factors in the design of the timeline: the ownership of the timeline in multi-user situations and the location of the detailed visual feedback resulting from interaction with the timeline. We use a collaborative digital tabletop board game to illustrate this design concept.
\end{abstract}

\section{Author Keywords}

tabletop display; gaming; automation; situation awareness; multi-user; interaction design;

\section{ACM Classification Keywords}

H5.2. User Interfaces; 


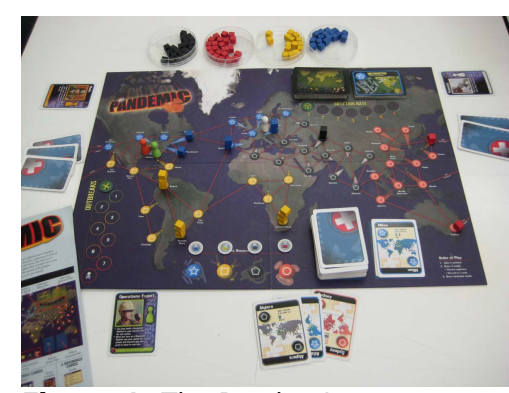

Figure 1: The Pandemic game requires 3 to 4 players to collaboratively collect a set of cures and save the world from epidemic outbreaks. The wayers lose if they run out of time players lose if they run out of time (cards they draw from) or when the game state gets out of control (too many diseases or outbreaks).

The game poses many challenges to the player and requires them to make many trade-offs, such as collecting cards that can be traded for cures (to win) vs. playing cards to keep the situation under control (to a control (to avoid losing).

\section{Introduction}

As digital tables become widely available, they are being designed to support complex tasks that require complex application behaviours, such as analyzing or simulating potential command and control or disaster management strategies $[4,5]$. High levels of automation are often required to extend the physical and cognitive limitations of human operators. Examples of automation include advancing military units and enforcing rules of engagement and combat in a simulation. It is essential that people using such systems have a high level of awareness of the situation and of the potential consequences of any changes occurring in the environment, which is often referred to as their level of situation awareness [2]. However, high levels of automation can leave people "out of the loop", where they do not understand how the system has changed, or why those changes occurred [1]. This can leave users unable to respond appropriately to critical system events. The problem of situation awareness has been observed in both traditional [2] and tabletop interfaces [8]. Tabletops both require and afford powerful solutions to this problem through their collaborative directlymanipulated nature.

The aim of this work is to improve situation awareness in a collaborative tabletop environment where actions are automated, so that groups can perceive changes in the system, comprehend them, predict future events, and, ultimately, make optimal decisions. We use strategic tabletop board games as a representative engaging, dynamic task scenario to investigate the effectiveness of potential design solutions. Digital tabletop board games allow us to rapidly prototype and evaluate potential designs. Moreover, this approach simplifies the process of recruiting "expert" users in and around a university campus, as many students play board games on a regular basis. Thus, realistic task behavior is likely to occur during the study, improving the validity of study results. Finally, previous research has shown similar, negative impacts of automation on situation awareness in digital tabletop board games consistent with automation research in other application areas [8]

Graphical interactive event timelines with the ability to replay past events have been previously shown to reduce response time and improve decision accuracy after interruptions in dynamic environments $[6,7]$. In this paper, we propose the use of similar interactive event timelines that support exploration and analysis of game history to improve situation awareness of both automated system actions and actions by players.

As work-in-progress, questions still remain regarding how to best design such an interactive event timeline. In particular, where should the historical event timeline be located and who should control it? In a collaborative photo-tagging task, Morris et al. [3] found that a shared centralized menu widget resulted in higher levels of collaboration, but that individual replicated menus were preferred by users. As our proposed timeline will be used to control the display of historical event data, providing a centralized, shared timeline versus replicated individual times may lead to different user preferences and task performance. Thus, location and ownership will be key design factors investigated in this research.

To contextualize our design concepts, we first describe the digital tabletop board game, called Pandemic ${ }^{1}$, we

${ }^{1}$ Published by Z-Man Games, used with permission. 


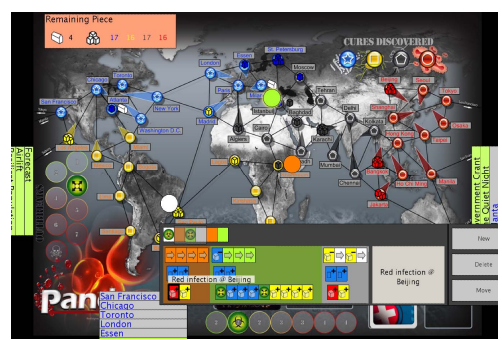
the digital game automates most of the game mechanics for the opponents (players used to carry these out manually). Also, it resolves special events that require several steps. Finally, it automates housekeeping and scorekeeping activities, such as shuffling decks, drawing cards, and updating counters. (The border is cropped out in this picture.)
Figure 2: Similar to a video game,

use as a case study for investigating tabletop automation (see Figure 1). Next, we describe our timeline design concepts and intended user study. Finally, we conclude and discuss future work.

\section{Pandemic and Automation}

Pandemic requires intense discussion and strategy formation as well as resource management. Throughout the game, critical events occur and the game situation becomes more chaotic. Thus, players require high levels of situation awareness to make correct decisions.

The digital version (Figure 2) automates the actions of the opponent (corresponding to the physical game board), as well as some complex activities. The automation reduces manual workload to allow players to spend more time discussing their strategies.

\section{Interactive Event Timeline}

We present an interactive event timeline, designed to improve players' situation awareness. Players can explore the game history, which consists of players' ac-

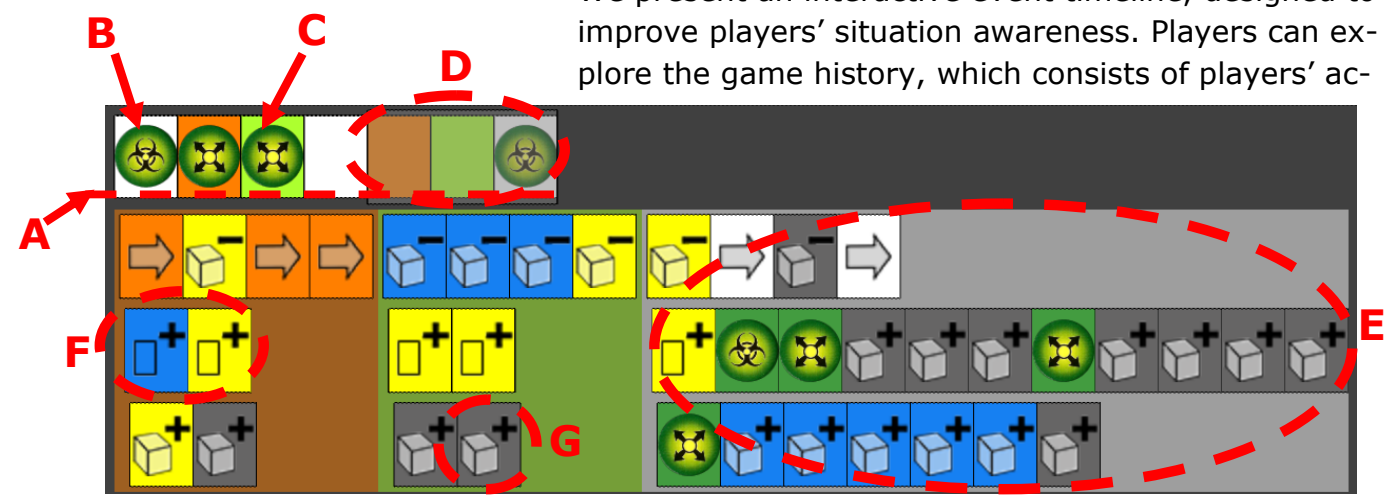

Figure 3: A close up of the awareness display. (A) overview bar composed of color blocks (player turns) with symbols denoting important events; (B) a special game event called epidemic; (C) a special game event named outbreak; (D) draggable viewport for navigating between player turns; (E) a player turn block which consists of three rows; (F) one phase in a player's turn; (G) an action block representing a player's or an automated action with symbols giving more details of the action (e.g., +/- for add/remove a game piece and arrow for a change in player location). tions and automation (see Figure 3). The timeline consists of two main components. The top bar provides a high-level overview of historical game events, while the bottom area shows turn-by-turn game history. The overview bar is used to select the specific turns to display in the detailed turn view.

The overview bar (Figure 3A) represents each player's turn, by color, with a symbol showing the key event occurred during that turn. Event symbols are used from the original Pandemic board game (Figure 3B and $3 \mathrm{C}$ ). The light grey viewport (Figure 3D) can be dragged to change which players' turn details are displayed in the detailed turn view below.

In the turn view, the game history is organized by player turn (Figure 3E). The three rows in each turn represent different game phases, making the phases easily distinguishable. Each row contains a series of action blocks (Figure 3G) that represent either a player's or an automated action. The colours correspond directly to Pandemic game-specific colour coding, and the symbols correspond to specific event types. Tapping on an action block provides more information. This event feedback can be visualized on the game board or within the timeline itself, as will be discussed below.

\section{Ownership and Location}

Two open questions regarding the design of the interactive event timelines relate to who can access the timeline (i.e. timeline ownership) and to where the event feedback should be provided when someone interacts with a timeline action block (feedback location).

Timelines can be shared between players or dedicated to each individual. A shared timeline, whose use must 


\begin{tabular}{|c|c|c|c|}
\hline & \multicolumn{2}{|c|}{ Ownership } \\
\hline & & Shared & Dedicated \\
\hline \multirow{3}{*}{ 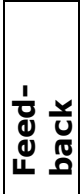 } & $\begin{array}{l}\text { Game } \\
\text { board }\end{array}$ & 1 & 4 \\
\hline & $\begin{array}{l}\text { Time- } \\
\text { line }\end{array}$ & 2 & 5 \\
\hline & Both & 3 & 6 \\
\hline
\end{tabular}

Table 1: Potential conditions based on different design factors.

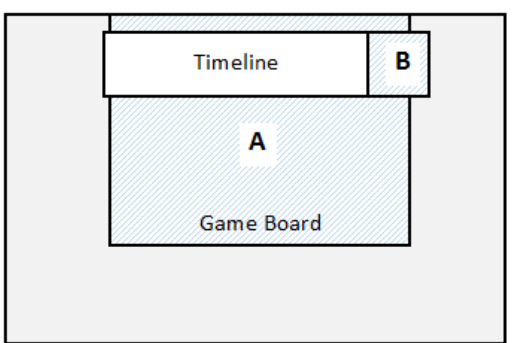

Figure 4: One shared timeline. (A) shared game board (the map). (B) space next to a timeline. Cond. 1 displays the game history feedback at $A$ only, Cond. 2 at $B$ at both $A$ and $B$.

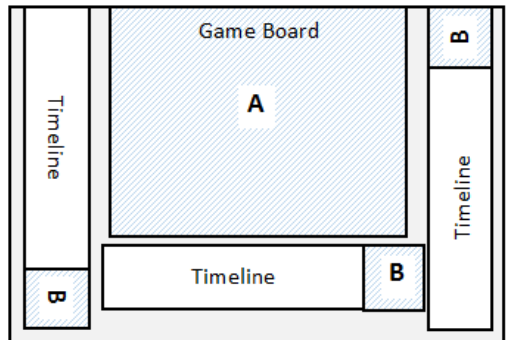

Figure 5: Dedicated timeline. (A) shared game board. (B) space adjacent to a timeline. For all timelines, Cond. 4 displays the feedback at $A$, Cond. 5 at the corresponding $B$, and Cond. 6 at both $A$ and $B$. be coordinated among players, may encourage discussion and provide a common reference point while working together. On the other hand, providing dedicated timelines, where each player has their own copy of the timeline, may better support independent work and allow players to investigate strategies separately. Both setups may improve situation awareness, as players can either learn from others through discussion or explore the game history themselves.

The event feedback can be placed on the timeline itself, on the game board area, or in both places. When placed on the timeline, an area adjacent to the turn view will be used to provide the game history. This combines the timeline and event information in one place. For feedback on the game board, the region that corresponds to the selected action block will be highlighted. This setup could easily facilitate player discussion, since it provides a reference point for all players. The feedback can also be placed both on the timeline and on the game board to provide more flexibility

\section{Planned Study}

The next step for this project is to conduct a user study to evaluate the impact of the different timeline design factors on players' situation awareness. The study wil consist of two phases. The first phase will use the factors mentioned previously, as summarized in Table 1. Figure 4 shows the sketches of Conditions 1 to 3, where there is only one timeline shared among all players. Figure 5 shows Conditions 4 to 6 , where each player has a dedicated timeline. The location of the event feedback varies between conditions. In the second phase, players will be given a configurable timeline that allows them to replicate the timeline and to select where the event feedback is displayed, as desired. Un- derstanding how players transition between design configurations will help determine player preferences and also the utility of different configurations at different stages of the game for supporting situation awareness.

\section{Acknowledgement}

The project is funded by NSERC, the NSERC SurfNet network, and the Canada EU Leif exchange program. We thank Matt Leacock, Z-Man Games, and Todor Doychev (digital game board graphics design).

\section{References}

[1] Endsley, M.R. and Kiris, E.O. The Out-of-the-Loop Performance Problem and Level of Control in Automation. The Journal of the Human Factors and Ergonomics Society 37, 2 (1995), 381-394.

[2] Endsley, M.R. Automation and Situation Awareness. In R. Parasuraman and M. Mouloua, eds., Automation and Human Performance: Theory and Applications. Lawrence Erlbaum, Mahwah, NJ, (1996), 163-181.

[3] Morris, M.R., Paepcke, A., Winograd, T., and

Stamberger, J. TeamTag: Exploring Centralized versus Replicated Controls for Co-located Tabletop Groupware. Proc. of CHI'06, (2006), 1273-1282.

[4] Paelke, V., Nebe, K., Geiger, C., Klompmaker, F., and Fischer, $\mathrm{H}$. Designing Multi-Modal Map-Based Interfaces for Disaster Management. Proc. of ACHI'12, (2012), 95-100.

[5] Qin, Y., Liu, J., Wu, C., and Shi, Y. uEmergency: A Collaborative System for Emergency Management on Very Large Tabletop. Ext. Abstract of ITS'12, (2012), 399-402. [6] Scott, S.D., Mercier, S., Cummings, M.L., and Wang, E. Assisting Interruption Recovery in Supervisory Control of

Multiple Uavs. Proc. of the Human Factors and Ergonomics Society 50th Annual Meeting, (2006), 699-703.

[7] Scott, S.D., Wan, J., Sasangohar, F., and Cummings, M.L. Mitigating Supervisory-level Interruptions in Mission Control Operations. Proc. of Applied Human Factors and Ergonomics, (2008)

[8] Wallace, J.R., Pape, J., Chang, Y.-L.B., et al. Exploring automation in digital tabletop board games. Proc. of the ACM 2012 conference on Computer Supported Cooperative Work Companion, (2012), 231-234. 